\title{
On Predicting Large Scale Fading Characteristics with the MR-FDPF Method
}

\author{
Meiling Luo*†, Nikolai Lebedev*, Guillaume Villemaud ${ }^{\dagger}$, Guillaume de la Roche ${ }^{\ddagger}$ \\ Jie Zhang* ${ }^{* \S}$ and Jean-Marie Gorce ${ }^{\dagger}$ \\ ${ }^{*}$ Ranplan Wireless Network Design Ltd. Suite 25, The Business Competitiveness Centre \\ Kimpton Road, LU2 0SX, Luton, United Kingdom \\ Email: meiling.luo@ ranplan.co.uk \\ ${ }^{\dagger}$ CITI Laboratory, Université de Lyon, INRIA, INSA-Lyon, CITI, F-69621, Villeurbanne, FRANCE \\ Email: jean-marie.gorce@insa-lyon.fr \\ ${ }^{\ddagger}$ Mindspeed Technologies, Sophia Antipolis, France \\ Email: guillaume.delaroche@mindspeed.com \\ ${ }^{\S}$ Department of Electronic and Electrical Engineering, University of Sheffield \\ Mappin Street, Sheffield, S1 3JD, United Kingdom \\ Email: jie.zhang@sheffield.ac.uk
}

\begin{abstract}
The MR-FDPF (Multi-Resolution Frequency Domain Partial Flow) method is proven to be a fast and efficient method to simulate radio wave propagation. It is a deterministic model which can provide an accurate radio coverage prediction. In reality, radio channels have the nature of randomness due to e.g. moving people or air flow. Thus they can not be rigorously simulated by a pure deterministic model. However, it is believed that some statistics can be extracted from deterministic models and these statistics can be very useful to describe radio channels in reality. In this paper, large scale fading statistical characteristics are extracted based on the MR-FDPF method. They are validated by comparison to both the theoretical result and measurement. The match also demonstrates that MR-FDPF is capable of simulating large scale fading.
\end{abstract}

\section{INTRODUCTION}

Since the radio propagation channel is the foundation of wireless communications, a good understanding of the radio channel is necessary for reliable system design [1]. For instance, an accurate knowledge of channel characteristics is required for cellular operators to optimize the coverage and maintain the interference at the lowest possible level.

Typically, the characteristics of the radio channel can be obtained by the following ways:

- Channel measurements: Conducting channel measurements is the most reliable way to know the radio channel, but it is not always possible to conduct extensive channel measurements for every radio system since measurement campaigns are usually time consuming and costly.

- Empirical channel models: Empirical models [1] are constructed either based on simplifying assumptions concerning the physical geometry of the propagation environments or based on a best fit to measurements data conducted in a typical environment. However, empirical models suffer from a lack of accuracy as they do not take into account specific propagation environments.
- Deterministic channel models: Deterministic channel models typically include ray optical methods [2] and finite difference methods [3]. They are based on the theory of electromagnetic propagation. Deterministic models are known for their relatively high accuracy since they intrinsically take into account the propagation environment. For that reason, they are also called site-specific models [4]. However, deterministic models have the disadvantage of high computational load.

Among the three, deterministic channel models are a good choice considering the trade off between accuracy and cost. Therefore, in this paper, the MR-FDPF method [5][6][7][8], a finite difference method, is chosen as the propagation model. The MR-FDPF method is proven to be fast and efficient in providing the radio coverage prediction. The provided coverage has a high level of accuracy because it takes into account the specific propagation environment, and also because of its high spatial resolution. Since the MR-FDPF method is a deterministic channel model, the signal strength provided by it is deterministic, but not stochastic. However, in reality radio channels have the nature of randomness due to e.g. moving people or air flow. Therefore, even while possessing the property of high accuracy, the MR-FDPF method can not describe the random aspect of radio channels. However, it is believed that some useful statistics, like large scale fading statistics, can be extracted from the deterministic model. In this paper, we investigate this point and validate it by both the theoretical result and measurement.

The rest of the paper is organized as follows. Section II presents the concept of large scale fading. Then, in section III large scale fading characteristics are extracted based on the MR-FDPF method, followed by experimental evaluation in section IV. Finally, conclusion is given in section V. 


\section{LARGE SCALE FADING CHARACTERISTICS}

When expressed in $\mathrm{dB}$, the instantaneous path loss can be considered as the sum of the mean path loss, shadow fading and small scale fading as follows [4]

$$
P L(d)=L(d)+X_{\sigma}+F
$$

where $P L(d), L(d), X_{\sigma}$ and $F$ denote the instantaneous path loss, the mean path loss, shadow fading and small scale fading associated with the Tx-Rx (transmitter and receiver) separation distance $d$, respectively. Unlike small scale fading which characterizes the rapid fluctuations of the received signal strength over very short travel distances (a few wavelengths), large scale fading, referring to the total effect combining the mean path loss and shadow fading shown in equation (1), characterizes signal strength variation over large distances [1].

Typically the mean path loss $L(d)$ is deterministic and is $\log$ dependent on the Tx-Rx separation distance $d$ as follows [1]

$$
L(d)=L_{0}+10 n \cdot \log _{10}(d)
$$

where $L_{0}$ is a constant which accounts for system losses and $n$ is the path loss exponent depending on the specific propagation environment. For instance in free space propagation $n=2$.

The shadow fading $X_{\sigma}$ is a zero-mean Gaussian distributed random variable (in $\mathrm{dB}$ ) with standard deviation $\sigma$. Small scale fading is typically described by Rayleigh distribution for NLOS (non-line-of-sight) propagation and Rice distribution for LOS (line-of-sight) propagation [1].

Large scale fading characteristics are very useful for determining the coverage area or evaluating system performance. For instance, with large scale fading characteristics, system designers can determine how large the coverage area of a transmitter is and what fade margin is required to achieve a certain level of edge reliability, e.g., 90\% edge reliability.

\section{EXTRACTION OF LARGE SCALE FADING CHARACTERISTICS BASED ON THE MR-FDPF METHOD}

In this section, a brief introduction of the MR-FDPF method is provided. We then investigate whether the MR-FDPF method is capable of simulating shadow fading phenomenon and how to extract fading characteristics based on the MRFDPF method.

\section{A. Multi-Resolution Frequency Domain Partial Flow method}

MR-FDPF method is the frequency domain version of (time domain) Partial Flow method [5]. The Partial Flow method is a numerical solution of Maxwell's equations based on the cellular automata formalism [9][10]. Detailed information about the Partial Flow method can be found in [11][12]. For the reason of simplification, the Partial Flow method is transfered into frequency domain by taking the Fourier transform. Moreover, the computational load of the frequency domain Partial Flow method can be further reduced by introducing the multi-resolution structure [5].

Considering the high computational load, most of the time MR-FDPF method is restricted to 2 dimensions (2D). This introduces an additional approximation error when compared to a real 3D propagation. Therefore, a calibration process seems to be imperative [6].

The calibration can be done in two steps. The first step is to estimate the constant offset as follows

$$
\Delta \Psi=\frac{1}{m} \sum_{k=0}^{m}\left(\Psi_{m e s}(k)-\Psi_{s i m}(k)\right)
$$

where $\Psi_{\text {mes }}(k)$ and $\Psi_{\text {sim }}(k)$ are the mean powers from measurements and simulations, respectively, and $m$ is the number of samples. A constant offset always exists because of the numerical sources used in the MR-FDPF method, compared to the real transmitters in reality. An attenuation coefficient of the air $\alpha_{\text {air }}$ is introduced by MR-FDPF in order to compensate the inaccuracy of the $2 \mathrm{D}$ model to simulate a real 3D propagation [13]. Besides, the set of parameters $\left(n_{\text {mat }}, \alpha_{\text {mat }}\right)$ of materials, where $n_{\text {mat }}$ and $\alpha_{\text {mat }}$ are the reflection factor and the attenuation coefficient of materials respectively, should also be calibrated since we never know exactly the material and the width of walls. Therefore, the second step of the calibration process is to estimate the $\alpha_{\text {air }}$ and $\left(n_{\text {mat }}, \alpha_{\text {mat }}\right)$ by minimizing the cost-function $Q$ defined by the root mean square error (RMSE) between measurements and predictions

$$
Q=R M S E=\sqrt{\frac{1}{m} \sum_{k=0}^{m}\left\|\Psi_{m e s}(k)-\Psi_{\text {pred }}(k)\right\|^{2}}
$$

where

$$
\Psi_{\text {pred }}(k)=\Psi_{\text {sim }}(k)+\Delta \Psi
$$

are the mean powers from predictions. The minimization process is solved by the direct search algorithm "DIRECT" by Jones et Al. in [14].

In Fig. 1, radio coverage provided by the MR-FDPF method is presented, obtained from a narrowband signal at $2.45 \mathrm{GHz}$ with $0 \mathrm{dBm}$ transmitter power.

\section{B. Capability of Simulating Shadow Fading Phenomenon}

From a theoretical point of view, small scale fading and shadow fading can be averaged out over an area, e.g. a square area, of a proper size. In other words, neither small scale fading nor shadow fading can be observed if the averaging area is large enough. The obtained path loss curve after averaging over a large enough area will be very smooth because it only contains the mean path loss.

If MR-FDPF is capable of simulating the fading characteristics, it should show the averaging effect described above. Therefore, we investigate this by doing averaging over square areas of different sizes, from $5 \lambda \times 5 \lambda$ to $50 \lambda \times 50 \lambda$, with a step of $5 \lambda$, where $\lambda$ denotes the wavelength. The averaging effect from MR-FDPF simulation is shown in Fig. 2. In this figure, a shadowing effect is evidenced over $5 \lambda \times 5 \lambda$ area, and $10 \lambda \times 10 \lambda$ area too, but there is a trend: the larger the size of the averaging area is, the less the shadowing effect can be observed. When the size of the area is about $40 \lambda \times 40 \lambda$, the shadowing effect almost can not be observed, which means that the shadowing has been averaged out.

Fig. 2 demonstrates that MR-FDPF is capable of providing the shadow fading phenomenon. 


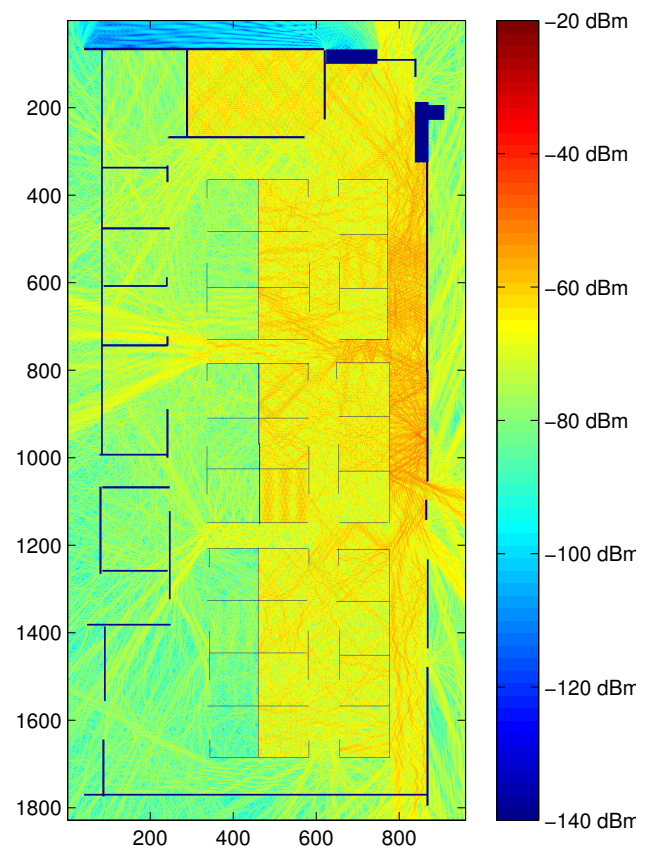

Fig. 1. radio coverage prediction provided by the MR-FDPF method at $2.45 \mathrm{GHz}$.

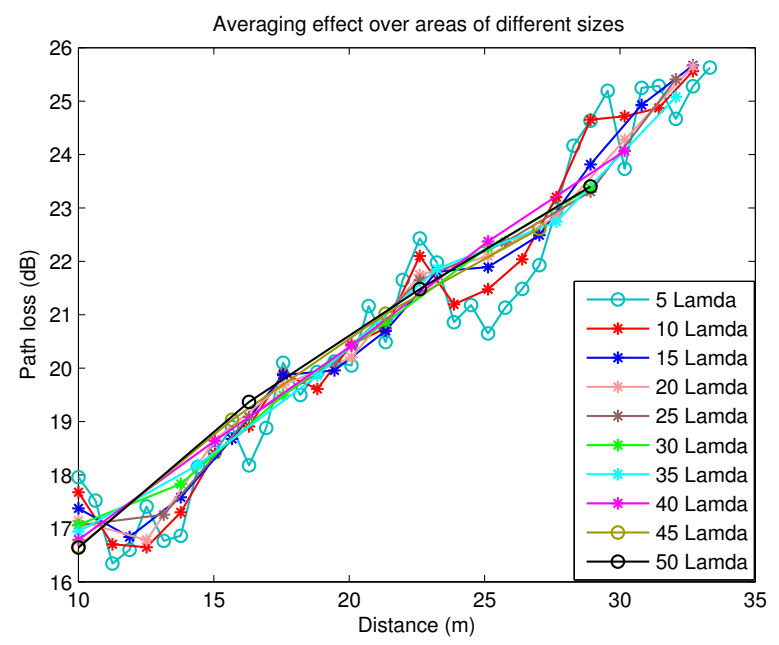

Fig. 2. The averaging effect over areas of different sizes.

\section{Extraction of Large Scale Fading Characteristics}

In this subsection, we describe the extraction of the large scale characteristics, namely the mean path loss and the shadow fading. In order to get the large scale characteristics, first we should average out the small scale fading. The obtained path loss after averaging out the small scale fading is usually called the local mean path loss. The most critical point for averaging out the small scale fading is the determination of the size of the averaging area.

According to [15][16], if the samples are expressed in $\mathrm{dB}$, the number of samples associated with $90 \%$ confidence inter-

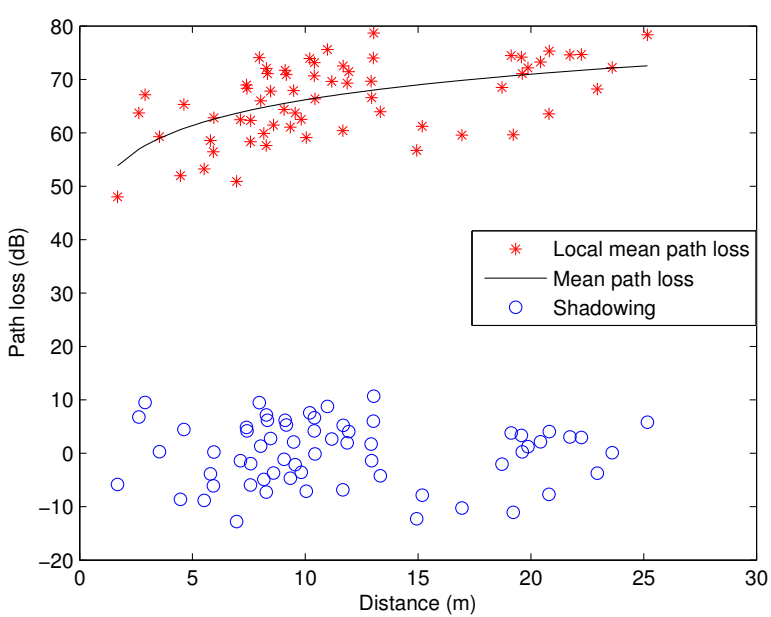

Fig. 3. The obtained local mean path loss, mean path loss and shadowing from the MR-FDPF method.

val is $N=85$. Provided that for MR-FDPF a square area can be chosen, the number of samples associated with each side of the square is thus $\sqrt{N}$. In addition, for a Rayleigh distributed signal envelope, the uncorrelated distance for two adjacent samples is $0.38 \lambda$ [16]. Finally, we obtain the minimum length of the side of the square is $0.38 \lambda \times \sqrt{N} \approx 3.8 \lambda$. On the other side, if it is a LOS propagation, the received signal envelope may be Rice distributed rather than Rayleigh, then in this case a smaller sample size may be sufficient [16].

Since the performance of MR-FDPF will be verified by comparison to the measurement conducted at Stanford by Dr. N. Czink [17] which will be described in section IV, we run MR-FDPF simulation with the same scenario as in the measurement. The simulation frequency is $2.45 \mathrm{GHz}$, and there are 8 transmitters and 8 receivers (i.e. 64 links in total). For more detailed scenario information, please refer to section IV.

According to the description above, the local mean path loss averaged over an area of $3.8 \lambda \times 3.8 \lambda$ is obtained shown in Fig. 3 by the red stars.

The local mean path loss includes both the mean path loss and the shadow fading. Since the mean path loss depends on the log distance shown in equation (2), we choose to get the $L_{0}$ and $n$ by using the curve fitting tool of Matlab such that the difference between the local mean path loss and the estimated mean path loss is minimized in a mean square error sense. Substituting the estimated $L_{0}=50.26$ and $n=1.592$ into equation (2), we obtain the mean path loss

$$
L(d)=50.26+10 \times 1.592 \cdot \log _{10}(d)
$$

Here $n=1.592$ indicates that there exists a waveguide effect in the propagation. The obtained mean path loss is shown by the black continuous curve in Fig. 3. Finally, the shadow fading can be obtained by just subtracting the mean path loss from the local mean path loss, which is denoted by the blue circles in Fig. 3.

In order to validate the shadow fading we have extracted, we compare its CDF (cumulative distribution function) with that of normal distribution. The comparison result is shown in 


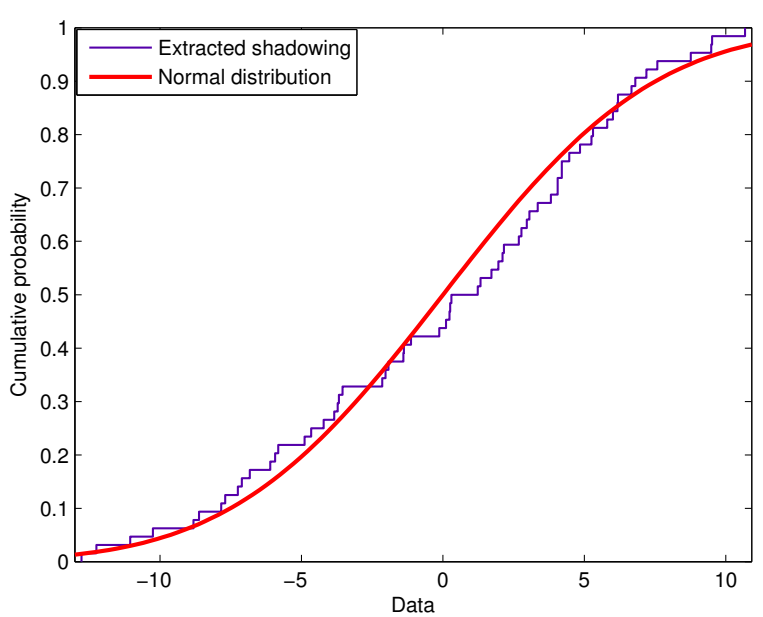

Fig. 4. CDF comparison between the extracted shadow fading from the MR-FDPF method and normal distribution.

Fig. 4. From the figure, we can see that the extracted shadow fading and the theoretical result are very similar.

Moreover, we also compute the standard deviation of the extracted shadow fading, which is $5.87 \mathrm{~dB}$.

\section{EXPERIMENTAL EVALUATION}

The results from the measurement campaign corresponding to "I2I moving receivers" scenario conducted at Stanford [17] are exploited to verify the performance of the proposed approach based on the MR-FDPF.

\section{A. The office scenario}

The scenario was a typical $16 \times 34 \mathrm{~m}$ office space made of 30 cubicles and 7 small separated rooms. 8 transmitters and 8 receivers were distributed in the office as illustrated in Fig. 5. All the transmitters were fixed in their locations, whereas all the receivers were randomly moved inside their cubicles when conducting the measurements. All the transmitters and receivers were equipped with omnidirectional antennas. Four materials were mainly used in the office, i.e., concrete for the main walls, plaster for the internal walls, glass for the external glass wall and wood for the cubicles located in the central part of the office.

\section{B. Measurement setup}

$8 \times 8$ MIMO channels at a center frequency of $2.45 \mathrm{GHz}$ were measured simultaneously with a RUSK MEDAV channel sounder [18]. In the measurement, 120 time blocks covering a total time of 32 seconds and 220 frequency bins covering a bandwidth of $70 \mathrm{MHz}$ were recorded. For large scale fading study, narrowband measurements are sufficient, hence the measurement data is restricted to the center frequency. Note that the averaging over the 120 time blocks implies the averaging over space since the receivers were moving when recording.

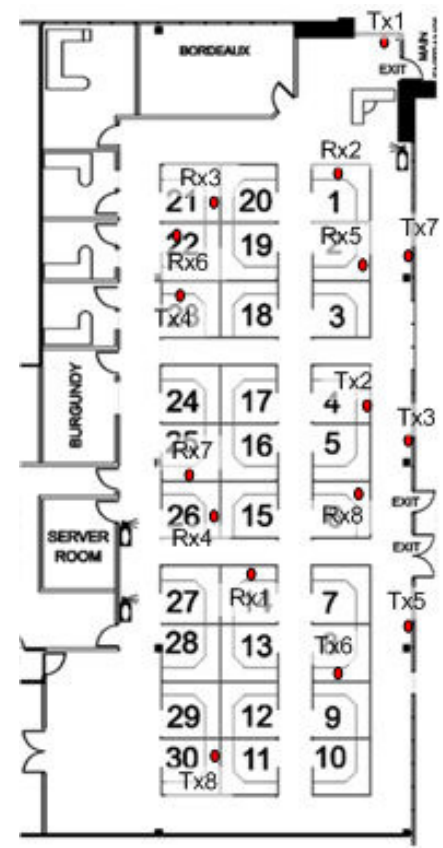

Fig. 5. The measurement scenario.

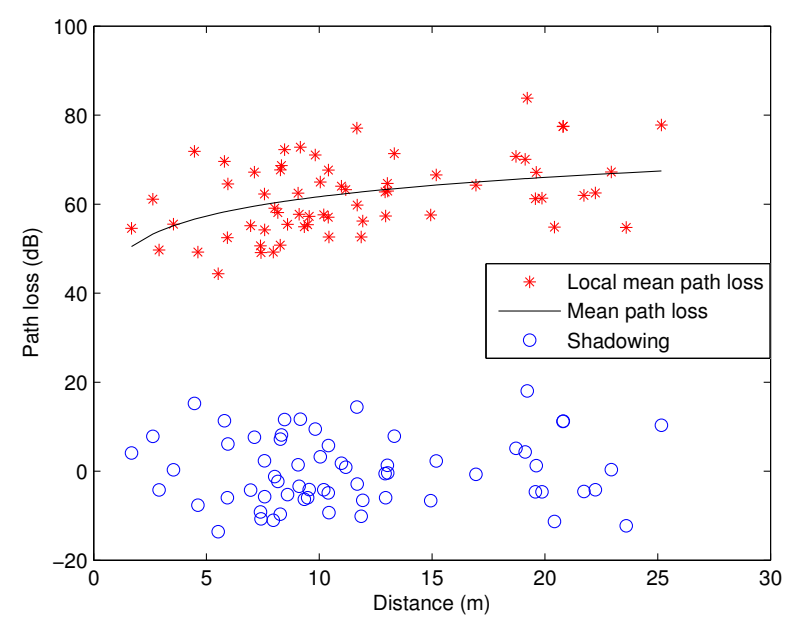

Fig. 6. The obtained local mean path loss, mean path loss and shadowing from measurement.

\section{Measurement results}

In this subsection, we extract large scale fading characteristics from the measurement. First of all, we obtain the local mean power by averaging over the 120 time blocks, which is shown by the red stars in Fig. 6. In the same way as in the simulation, the deterministic mean path loss is obtained by curve fitting

$$
L(d)=47.25+10 \times 1.442 \cdot \log _{10}(d)
$$

The mean path loss is shown in Fig. 6 by the black continuous curve. Once we get the mean path loss, the shadow fading is obtained by subtracting the mean path loss from the local mean path loss, which is denoted by the blue circles in Fig. 6.

The CDF comparison between the extracted shadow fading and normal distribution is shown in Fig. 7. This good match 


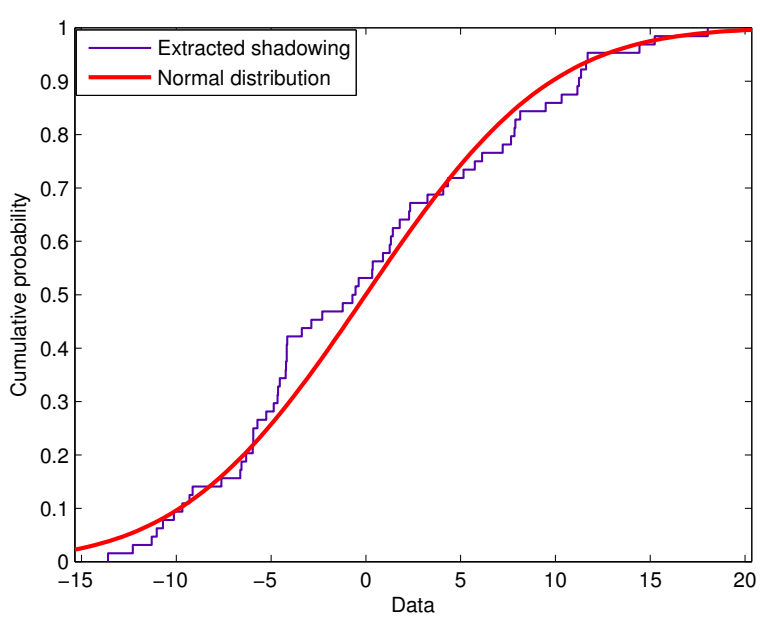

Fig. 7. CDF comparison of the extracted shadowing and normal distribution from measurement.

TABLE I

COMPARISON BETWEEN THE SIMULATION AND MEASUREMENT

\begin{tabular}{|c|c|c|}
\hline & $\mathrm{n}$ & The standard deviation $\sigma$ \\
\hline MR-FDPF & 1.59 & $5.87 \mathrm{~dB}$ \\
\hline Measurement & 1.44 & $7.66 \mathrm{~dB}$ \\
\hline
\end{tabular}

demonstrates that the proposed approach for extracting the shadow fading is effective. The standard deviation of the extracted shadow fading from the measurement is $7.66 \mathrm{~dB}$.

\section{Match between the simulation and measurement}

Compare the parameters extracted from the MR-FDPF method and measurement in Tab. I, we can see that they fit each other very well. The path loss exponents $n$ both from the MR-FDPF method and measurement are smaller than 2, which indicates that there exists a waveguide effect in the propagation. The standard deviation of shadowing from simulation is slightly lower than that from measurement. We suggest that this is due to the unmodeled furniture which would contribute more to fading. The match between the simulation and measurement demonstrates that the MR-FDPF method is capable of simulating large scale fading characteristics.

\section{CONCLUSION AND PERSPECTIVES}

Although deterministic channel models possess the advantage of high accuracy due to their ability to take into account the specific propagation environment, they can not rigorously simulate realistic radio channels presenting random phenomenon. However, some useful statistics can be extracted from deterministic channel models. Basically, deterministic channel models combine all propagation phenomenons, i.e., the deterministic mean path loss, shadow fading and small scale fading. Thus, in order to extract fading statistics, we should first try to separate them. In this paper, large scale fading characteristics are extracted based on the MR-FDPF method. The extracted fading characteristics are validated by comparison to both the theoretical results and measurement.
The match demonstrates that MR-FDPF method is capable of simulating large scale fading characteristics.

In the future, more measurements would be conducted in order to validate the extraction approach based on MRFDPF in other scenarios and/or frequency bands. Furthermore, although this paper is mainly based on the MR-FDPF method, it is worth investigating whether our approach can be applied to other deterministic channel models, e.g. ray tracing models to extract the fading statistics.

\section{ACKNOWLEDGMENT}

The authors would like to thank Dr. Nicolai Czink from FTW of Vienna for performing the measurements and providing the data. This work is funded by the FP7 IPLAN Project.

\section{REFERENCES}

[1] T. Rappaport et al., Wireless communications: principles and practice. Prentice Hall PTR New Jersey, 1996, vol. 207.

[2] C. Balanis, Advanced Engineering Electromagnetics. New York, NY, USA: John Wiley \& Sons, 1999.

[3] K. Yee, "Numerical solution of initial boundary value problems involving maxwell's equations in isotropic media," IEEE Transactions on Antennas and Propagation, vol. 14, no. 3, pp. 302-307, 1966.

[4] A. Molisch, Wireless communications. New York, NY, USA: John Wiley \& Sons, 2011.

[5] J.-M. Gorce, K. Jaffres-Runser, and G. de la Roche, "Deterministic approach for fast simulations of indoor radio wave propagation," IEEE Transactions on Antennas and Propagation, vol. 55, no. 3, pp. 938 -948, 2007.

[6] K. Runser and J.-M. Gorce, "Assessment of a new indoor propagation prediction method based on a multi-resolution algorithm," in IEEE 61st Vehicular Technology Conference, VTC 2005-Spring, vol. 1, 2005, pp. 35 - 38 Vol. 1.

[7] M. Luo, D. Umansky, G. Villemaud, M. Lafort, and J.-M. Gorce, "Estimating channel fading statistics based on radio wave propagation predicted with deterministic mrfdpf method," in 5th European Conference on Antennas and Propagation (EuCAP 2011), Rome, Italy, April, 2011.

[8] M. Luo, G. De La Roche, G. Villemaud, J. Gorce, D. Umansky, J. Zhang et al., "Simulation of wide band multipath fast fading based on finite difference method," in IEEE Vehicular Technology Conference, no. 2011, 2011.

[9] B. Chopard, P. Luthi, and J.-F. Wagen, "Lattice boltzmann method for wave propagation in urban microcells," IEE Proceedings - Microwaves, Antennas and Propagation, vol. 144, no. 4, pp. 251 -255, Aug. 1997.

[10] B. Chopard and M. Droz, Cellular automata modeling of physical systems. Cambridge University Press, 1998.

[11] P. Luthi, "Lattice wave automata: from radio wave to fracture propagation," Ph.D. dissertation, $\mathrm{PhD}$ thesis, Computer Science Department, University of Geneva, 24 rue General-Dufour, 1211 Geneva 4, Switzerland, 1998.

[12] B. Chopard and P. Luthi, "Lattice boltzmann computations and applications to physics," Theoretical Computer Science, vol. 217, no. 1, pp. 115-130, 1999.

[13] G. de la Roche, K. Jaffres-Runser, and J.-M. Gorce, "On predicting in-building wifi coverage with a fast discrete approach," International Journal of Mobile Network Design and Innovation, vol. 2, pp. 3-12, 2007.

[14] D. Jones, C. Perttunen, and B. Stuckman, "Lipschitzian optimization without the lipschitz constant," Journal of Optimization Theory and Applications, vol. 79, no. 1, pp. 157-181, 1993.

[15] S. Saunders and A. Aragón-Zavala, Antennas and propagation for wireless communication systems. Wiley, 2007.

[16] J. Parsons, The mobile radio propagation channel. Wiley Online Library, 2000, vol. 81.

[17] N. Czink, B. Bandemer, G. Vazquez-Vilar, A. Paulraj, and L. Jalloul, "July 2008 radio measurement campaign: Measurement documentation," Stanford University, Smart Antennas Research Group, Tech. Rep., July 2008.

[18] "Rusk medav channel sounders," 2008, [Online]. Available: http://www.channelsounder.de. 